\title{
Flexicurity und atypische Beschäftigung in Dänemark
}

\author{
Thomas Bredgaard, Flemming Larsen, \\ Per Kongshøj Madsen, Stine Rasmussen
}

\begin{abstract}
Das Verhältnis von atypischer Beschäftigung und Flexicurity in Dänemark ist bisher nicht detailliert untersucht worden. Das ist wahrscheinlich auf die weit verbreitete Annahme zurückzuführen, dass die Nutzung von atypischen Beschäftigungsverhältnissen in Dänemark vergleichsweise geringfügig sei und diese darüber hinaus schon gut geschützt wären. Da die Beschäftigungsmobilität der normalen Arbeitnehmerschaft hoch sei - so die weitere Vermutung - müssten dänische Arbeitgeber nicht auf „atypische“ Beschäftigungsverhältnisse ausweichen, um ihr Bedürfnis nach extern-numerischer Flexibilität zu befriedigen. Ein genauerer Blick auf die Absicherung atypischer Beschäftigung legt jedoch weitere Erklärungen nahe.
\end{abstract}

\section{Einleitung}

Flexicurity - die begriffliche Verknüpfung von flexibility und security - ist in einer bemerkenswert kurzen Zeit zur politischen Attraktion geworden (Jørgensen/Madsen 2007). Flexicurity gilt zurzeit als das gefragteste Konzept für Reformen der Arbeitsmärkte, der Arbeitsgesetzgebung und der Beschäftigungspolitik in der Europäischen Union (EU). Die EU-Kommission definiert Flexicurity dabei eher wage als eine integrative Strategie, um gleichzeitig die Flexibilität und die soziale Sicherheit auf dem Arbeitsmarkt zu erhöhen (European Commission 2007a, S. 4).

Dänemark gilt als Paradebeispiel für den Flexicurity-Ansatz. Hier wird ein flexibler Arbeitsmarkt mit einer großzügigen sozialen Absicherung und einer aktiven Arbeitsmarktpolitik kombiniert - eine Verbindung, die auch unter den Namen „goldenes Dreieck" bekannt geworden ist (OECD 2004; Ministry of Labour 1999).

In der Literatur wird der dänische Flexicurity-Ansatz häufig als ein Arbeitsmarktmodell aufgefasst, das auf den regulären Arbeitsmarkt für Normalarbeitsverhältnisse zielt (Madsen 2003, 2004; Bredgaard et al. 2005). Eines der Hauptmerkmale des dänischen Beschäftigungssystems ist der relativ niedrige Kündigungsschutz (OECD 2004, Abschnitt 2). Aus diesem Grund verzichten die dänischen Arbeitgeber bisher weitgehend auf den Einsatz von atypisch Beschäftigten. Vereinfacht ausgedrückt können die Normalbeschäftigten aufgrund ihrer großen beruflichen Mobilität und hohen Fluktuation als zeitlich befristete Arbeitnehmer bezeichnet werden (Bredgaard et al. 2005, 2006).

Das Arbeitslosenversicherungssystem dient zudem als flexibilisierungsförderndes Mittel, das die Mobilität und die Risikofreude der Arbeitnehmer fördert. Für diejenigen, die Schwierigkeiten haben, eine neue Anstellung zu finden, soll im Idealfall außerdem die aktive Arbeitsmarktpolitik dazu beitragen, die Qualifikation und Motivation zu steigern und so Chancen zur Reintegration in den Arbeitsmarkt zu schaffen (Madsen 2003, 2004, 2005, 2006).

Dieses Bild ist jedoch nicht präzise genug. Im Folgenden analysieren wir deshalb das Verhältnis zwischen dänischer Flexicurity und atypischer Beschäftigung. Zunächst untersuchen wir dabei den Zusammenhang zwischen Kündigungsschutz und atypischer Beschäftigung (Abschnitt 2). In einem zweiten Schritt beschreiben wir die Verbreitung, Entwicklung und Regulierung dieser ,atypischen“ Beschäftigungsverhältnisse in Dänemark (Abschnitt 3) und stellen zum Schluss die komplexen Zusammenhänge zwischen Flexicurity und atypischen Beschäftigungsformen in Dänemark dar (Anschnitt 4).

Unter atypischer Beschäftigung verstehen wir die Haupttypen nicht standardisierter Beschäftigungsformen, die von einer unbefristeten abhängigen Vollzeitbeschäftigung bei einem Arbeitgeber abweichen. Wenn atypische Beschäftigung in diesem Sinne als Gegensatz zur standardisierten Beschäftigung aufgefasst wird, kann sie auch Werkverträge, Heimarbeit, Telearbeit, verschiedene flexible Arbeitszeitmodelle, Job-Sharing, Nebenverdienste, Saisonar- beit, Schwarzarbeit, Familienarbeit usw. einschließen (Kalleberg 2000, S. 343f.). Wir bleiben hier jedoch bei der konventionellen europäischen Definition von atypischer Beschäftigung, die sich eher auf die bedeutendsten Gruppen nicht standardisierter Beschäftigung bezieht: Teilzeitarbeit, Befristung, Leiharbeit und Selbstständigkeit. Als eine besondere dänische Ergänzung haben wir auch die wachsende Zahl der sogenannten Flexjobs einbezogen; das sind Arbeitsplätze mit auf Dauer gewährten Lohnzuschüssen, die Personen mit eingeschränkter Arbeitsfähigkeit vorbehalten sind.

Thomas Bredgaard, Associate Professor, Ph.D., Centre for Labour Market Research at Aalborg University (CARMA). Arbeitsschwerpunkte: Aktive Arbeitsmarktpolitik, Flexicurity, Corporate Social Responsibility e-mail: thomas@epa.aau.dk Flemming Larsen, Associate Professor, CARMA, Arbeitsschwerpunkte: Aktive Arbeitsmarktpolitik, Flexicurity, Wirkungsforschung.

e-mail: flemlar@epa.aau.dk Per Kongshøj Madsen, Professor, CARMA, Arbeitsschwerpunkte: Aktive Arbeitsmarktpolitik, Flexicurity, Wirkungsforschung. e-mail: pkm@epa.aau.dk Stine Rasmussen, Research Assistant, CARMA, Arbeitsschwerpunkte: Atypische Beschäftigung, Flexicurity, Arbeitslosenversicherungssysteme. e-mail: sra@epa.aau.dk

Übersetzung aus dem Englischen von Karin Vitols 


\section{Kündigungsschutz und "atypische" Beschäftigung}

Diskutiert man den Zusammenhang zwischen der gesetzlichen Regelung des Kündigungsschutzes und der Verbreitung von atypischer Beschäftigung in Dänemark, so begegnet man einigen gängigen Annahmen: Im Zeitalter raschen technologischen Wandels, internationalisierter Arbeitsmärkte und individualisierter Lebensentwürfe gehöre das stabile Langzeitbeschäftigungsverhältnis der Vergangenheit an. Ein strenger Kündigungsschutz fördere (auch wegen der veränderten Bedingungen) atypische Beschäftigung. Folglich sei ein geringerer Kündigungsschutz ein Weg zur Vermeidung segmentierter Arbeitsmärkte. Da der dänische Arbeitsmarkt von Normalarbeitsverhältnissen und ihm äquivalenten Beschäftigungsformen geprägt ist, wird daraus geschlussfolgert, dass der in Dänemark schwach ausgeprägte Kündigungsschutz die Dominanz des Normalarbeitsverhältnisses auch in der heutigen Zeit erklärt. Bei näherem Hinsehen muss man dieses Bild jedoch modifizieren.

Die erste Annahme inspirierte zur Umorientierung der sozialen Absicherung weg von der Arbeitsplatzsicherheit (Kündigungsschutz) hin zur Beschäftigungssicherheit im Sinne von Förderung der Qualifikation und verbesserter Vermittlung in Arbeit sowie finanzieller Kompensation bei Übergängen. Es liegen allerdings kaum empirische Beleg dafür vor, dass sichere Arbeitsplätze in Dänemark ein Auslaufmodell sind. Im Gegenteil: Schon Auer/Cazes (2003) stellten eine überraschend hohe Stabilität der Beschäftigungsverhältnisse im Zeitverlauf fest. Die durchschnittliche Beschäftigungsdauer hat sich in der Zeit von 1992 bis 2005 kaum verändert (Auer 2007).

Die zweite Annahme unterschlägt zunächst, dass ein strenger Kündigungsschutz für Arbeitnehmer in Normalarbeitsverhältnissen durch verschiedene Arten der Flexibilität aufgefangen werden kann, um die Produktivität und Wettbewerbsfähigkeit zu erhalten. Nicht nur externe Flexibilisierung (durch Arbeitnehmer in nicht standardisierten Beschäftigungsverhältnissen), auch interne Flexibilisierung (funktionale Flexibilität, Flexibilisierung der Arbeitszeiten oder Lohnflexibilität) ist denkbar. Trotzdem ergibt die Analyse des OECD-Indika-

\section{Abb. 1: Stärke der Arbeitsmarktregulierung (EPL) und Verbreitung von befristeten Arbeitsverträgen 2003}

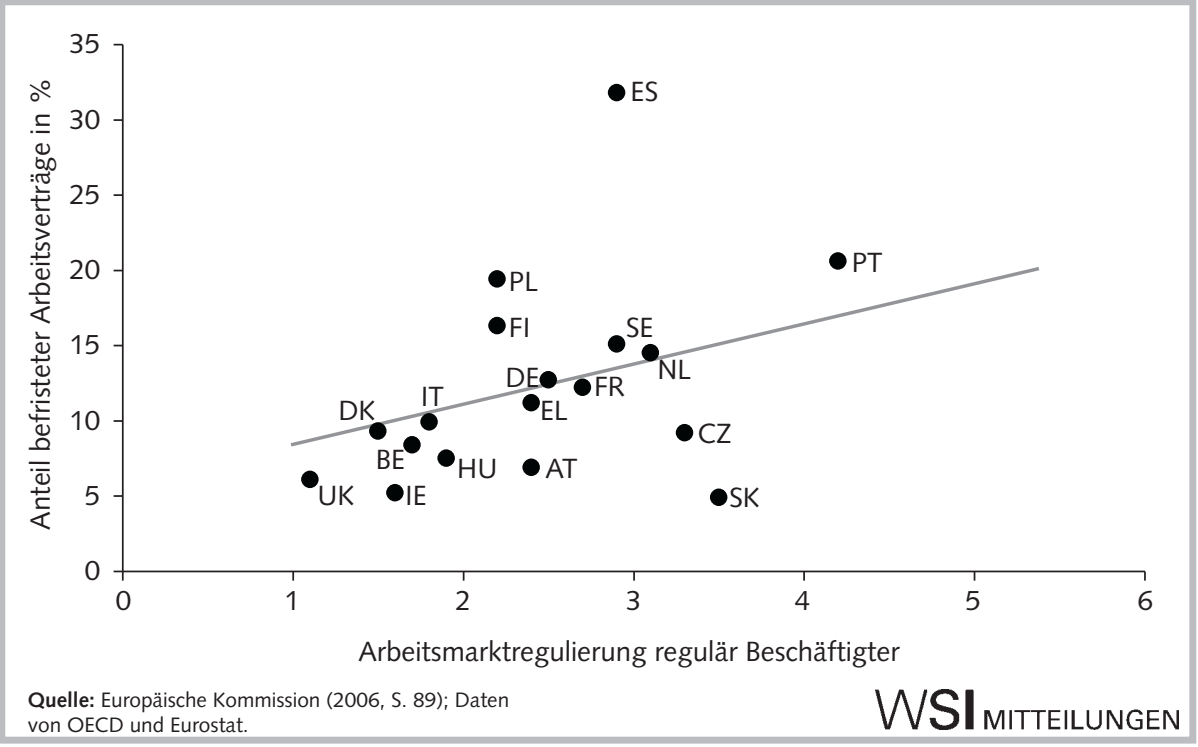

tors zur Stärke der Arbeitsmarktregulierung (EPL) in Normalarbeitsverhältnissen und des Eurostat Indikators zum Anteil der Arbeitnehmer mit befristeten Verträgen, dass eine strenge Regulierung von Normalarbeitsverträgen zu einem erhöhten Aufkommen von befristeter Beschäftigung beiträgt (Abbildung 1).

Wie erwartet, befinden sich die Länder mit einem geringeren Kündigungsschutz (wie Dänemark und Großbritannien) in der unteren linken Seite der Abbildung, während Länder mit strengerem Schutz (wie Portugal und Spanien) in der oben rechten Hälfte liegen. Gleichwohl ist die statistische Beziehung (= Steigung der Geraden) zwischen der Stärke der Arbeitsmarktregulierung und dem Anteil der Arbeitnehmerschaft in befristeten Arbeitsverhältnissen nur schwach ausgeprägt. Die Europäische Kommission verweist darauf, dass die partielle Einschränkung des Kündigungsschutzes (wie im Fall von befristet Beschäftigten) einen dualen Arbeitsmarkt mit ungesicherten befristeten Arbeitsplätzen und sicherer, aber unflexibler regulärer Beschäftigung schaffen könnte (European Commission 2006, S. 90).

Die dritte Annahme - Dominanz des Normalarbeitsverhältnisses und äquivalenter Formen bei geringer Verbreitung atypischer Beschäftigung - wird im Folgenden in zweierlei Hinsicht untersucht. Einerseits stellen wir die Verbreitung atypischer Formen dar, andererseits nehmen wir die Einbindung atypischer Beschäftigung in die dänische Flexicurity in den Blick. Denn es gilt zu klären, ob die Absicherung der vor- handenen atypischen Beschäftigung als dem Normalarbeitsverhältnis äquivalent angesehen werden kann.

\section{Atypische Beschäftigung in Dänemark}

Wie stellt sich die atypische Beschäftigung quantitativ dar? Auffällig für Dänemark ist die bemerkenswerte Stabilität des Anteils der Atypischen an der Gesamtbeschäftigung im Zeitverlauf: Teilzeit, Befristung und Selbstständigkeit blieben seit Mitte der 1990er Jahre fast auf dem gleichen Niveau, während Teilzeit und Befristung im EU-15Durchschnitt erheblich zunahmen. Die Selbstständigenquote ging in Dänemark wie in der EU-15 leicht zurück (European Commission 2007b, S. 286). Der Anteil der Flexjobs, eine dänische Besonderheit, stieg seit ihrer Einführung stark an; im Jahr 2006 lag ihr Anteil bei 1,7 \% der Erwerbstätigen.

Im Vergleich zu den Ländern der EU15 gibt es in Dänemark zwar ein viel niedrigeres Aufkommen von Selbstständigkeit (6 \% in Dänemark und $15 \%$ in der EU-15) und weniger Befristung ( $9 \%$ in Dänemark und $15 \%$ in der EU-15), aber der Anteil von Teilzeitbeschäftigung liegt in Dänemark (24\%) höher als in der EU-15 (21\%). Wie auch in anderen EU-Mitgliedsländern gibt es darüber hinaus bedeutende geschlechtsspezifische Unterschiede in der Verteilung von „atypischen“ Beschäftigungsverhältnissen. Im Jahr 2006 waren 
$35 \%$ aller weiblichen Beschäftigten in Teilzeit tätig (im Vergleich zu $13 \%$ der Männer) und nur $4 \%$ der Frauen waren selbstständig (im Vergleich zu $8 \%$ der Männer) (European Commission 2007b, S. 290).

Im folgenden Teil analysieren wir die gesetzliche Regulierung der verschiedenen Formen atypischer Beschäftigung in Dänemark. Mit Bezug auf die Standards in Normalarbeitsverhältnissen definieren wir Unsicherheit in Anlehnung an die FlexicurityMatrix (Wilthagen 1998; Wilthagen/Tros 2004) als ein geringeres Ausmaß an Arbeitsmarktregulierungen (EPL), sozialer Sicherheit, Beschäftigungsschutz (Beschäftigungsfähigkeit) und Absicherung der Vereinbarkeit von Arbeit und Leben (WorkLife Balance). So können wir die Frage beantworten, ob Personen in atypischen Beschäftigungsverhältnissen das gleiche $\mathrm{Ni}$ veau des sozialen Schutzes genießen, wie regulär Beschäftigte. Wir untersuchen hauptsächlich die formale Ebene des Schutzes in Rahmen von Gesetzen und Kollektivvereinbarungen ${ }^{1}$; die Umsetzung dieser Regulierung nur, wenn empirische Erkenntnisse vorliegen.

\subsection{TEILZEITARBEIT}

Nach Angaben der in Dublin ansässigen Europäischen Stiftung zur Verbesserung der Arbeits- und Lebensbedingungen legen empirische Befunde nahe, dass Teilzeitarbeit in Europa insgesamt mit schlechteren Arbeitsbedingungen als Vollzeitarbeit einhergeht. Teilzeitbeschäftigte geben an, weniger Möglichkeiten zu haben, an Weiterbildungen teilzunehmen und ihre Laufbahnentwicklung zu beeinflussen. Sie weisen eine niedrigere Beschäftigungsdauer, geringere Lohn- und Gehaltsniveaus, einen eingeschränkten Zugang zu Zusatzleistungen und zu Leistungen der sozialen Sicherung auf. Auf der anderen Seite geben Teilzeitbeschäftigte aber auch weniger berufsbezogene Gesundheitsprobleme an und erreichen häufiger ein ausgewogeneres Verhältnis von Arbeit und Leben (Work-Life Balance). Zwischen den Ländern existieren diesbezüglich jedoch erhebliche Unterschiede (Eurofound 2003, S. 2).

In Dänemark ist Teilzeitarbeit ebenso weit verbreitet wie konstant. Sie wird nicht als atypische oder prekäre Arbeit aufgefasst, sondern eher als normale Beschäftigungsform mit kürzeren Arbeitszeiten (Madsen/ Petersen 2000, S. 61). Wie in anderen europäischen Ländern ist Teilzeitarbeit in be- stimmten Sektoren überrepräsentiert (insbesondere Handel, Hotel- und Gaststättengewerbe und öffentlicher Dienst). Diese Konzentration bewirkt auch, dass ein höherer Anteil der Teilzeitbeschäftigten einen niedrigeren sozio-ökonomischen Status aufweist (Wehner et al. 2002, S. 10).

Das heißt jedoch nicht, dass alle Teilzeitbeschäftigten freiwillig in dieser Beschäftigungsform arbeiten. Im Jahr 2002 gaben $16 \%$ aller Teilzeitarbeiter in Dänemark im Labour Force Survey als Grund für ihre Teilzeitarbeit an, dass sie keine Vollzeitstelle finden konnten. Der Anteil unfreiwilliger Teilzeitbeschäftigung lag damit über dem EU-Durchschnitt von $14 \%$, aber weit unter den Anteilen, die für Griechenland (44\%), Finnland (31\%), Italien (31\%), Frankreich (24\%) und Schweden (22\%) berichtet werden (Eurofound 2003, S. 9). Ungefähr die Hälfte aller Teilzeitbeschäftigten in Dänemark gab an, dass der Hauptgrund für ihre Teilzeitarbeit darin besteht, dass sie nicht Vollzeit arbeiten wollen. Zusätzlich arbeiten ungefähr $30 \%$ in Teilzeit, weil sie in Aus- und Weiterbildungsaktivitäten eingebunden sind (wie beispielsweise Studenten). Folglich kann $80 \%$ der Teilzeitbeschäftigung als freiwillig bezeichnet werden. Die Motive unterscheiden sich nach Geschlecht: 60 \% der Männer arbeiten in Teilzeit, weil sie Arbeit und Bildung kombinieren wollen, während 58 \% der Frauen keine Vollzeitstelle haben möchten, unter anderem um die WorkLife Balance zu verbessern (Wehner et al. 2002, S. 4).

Die Regulierung der Teilzeitarbeit beruht auf Kollektivvereinbarungen und Gesetzen. Zum Beispiel wurde im Jahr 2001 die EU-Richtlinie zur Teilzeitarbeit aus dem Jahr 1997 für die Privatwirtschaft von den Sozialpartnern umgesetzt. Ergänzende Vereinbarungen wurden auch zwischen den Sozialpartnern, die verschiedene Beschäftigungsgruppen im öffentlichen Dienst vertreten, abgeschlossen. Das wichtigste Ziel war, eine unterschiedliche Behandlung von Vollzeit- und Teilzeitbeschäftigten zu verhindern, außer wenn objektive Gründe hierfür sprechen.

Im Jahr 2002 wurde von der liberalkonservativen Regierung ein neues Teilzeitgesetz erlassen. Das Gesetz stieß bei den Gewerkschaften auf Kritik: Zum einen sah man es als Einmischung in das traditionelle dänische Modell der freiwilligen Kollektivverhandlungen, zum anderen fürchtete man, dass Arbeitnehmer mit Vollzeitstel- len zur Aufnahme von Teilzeitarbeit gezwungen werden könnten. Das Teilzeitgesetz verfolgte allerdings eher die Absicht, Hindernisse, die in Kollektivvereinbarungen für die Teilzeitarbeit bestanden, auszuräumen. Nun haben Arbeitnehmer bei Zustimmung des Arbeitgebers die Möglichkeit, von Vollzeit auf Teilzeit zu wechseln. Außerdem muss der Arbeitgeber eine Entschädigung zahlen, wenn ein Arbeitnehmer aufgrund seiner Arbeitszeitwünsche entlassen wurde, sei es die Weigerung oder die Absicht, Teilzeit zu arbeiten. Folglich garantiert das Gesetz nicht dem Arbeitnehmer ein Recht auf Teilzeit, sondern bietet sie nur als Option an, wenn der Arbeitgeber zustimmt. Das Gesetz gilt nur für bereits Beschäftigte, nicht aber für Neueinstellungen. Hier gibt es weiterhin einige Hindernisse für Teilzeitarbeit, wie etwa eine zahlenmäßige Begrenzung der Teilzeitkräfte, die Regelung, dass Teilzeitarbeit nicht Vollzeitarbeit substituieren darf und dass Teilzeitbeschäftigung mit den gewerkschaftlichen Interessenvertretern im Betrieb (Shop Stewards) ${ }^{2}$ abgestimmt werden muss (Danish Confederation of Trade Unions 2004). Seit das Gesetz 2002 in Kraft getreten ist, hat die Verbreitung von Teilzeitarbeit um vier Prozentpunkte zugenommen.

Werden Teilzeitbeschäftigte arbeitslos, haben sie den gleichen Einkommensschutz wie Vollzeitbeschäftigte. Beide können Mitglieder eines Arbeitslosenversicherungsfonds werden. Eine Teilzeitversicherung ist für Personen verfügbar, die weniger als 30 Stunden pro Woche arbeiten. Die Beiträge und die Versicherungsleistungen sind für Teilzeitbeschäftigte niedriger. Die Leistungen dürfen zwei Drittel der Leistungen für

\footnotetext{
Die Regulierung von Beschäftigungsverhältnissen erfolgt auf drei Wegen: auf Ebene der Europäischen Kommission durch Richtlinien, auf Ebene der Nationalstaaten entweder durch Gesetze oder Kollektivvereinbarungen der Tarifparteien. In Dänemark war strittig, ob Kollektivvereinbarungen ausreichen, um EU-Richtlinien für alle verbindlich umzusetzen. Mittlerweile setzen die Sozialpartner die Richtlinien in Kollektivvereinbarungen um und die nationale Arbeitsgesetzgebung ergänzt dies gesetzlich so, dass auch solche Arbeitgeber und Arbeitnehmer abgedeckt sind, die nicht unter die Kollektivvereinbarungen fallen (erga omnes). Das langfristige Überleben dieses Umsetzungsmodells ist jedoch noch immer unklar (Nedergaard 2004).

2 Zur betrieblichen Vertretung von Arbeitnehmerinteressen im sogenannten Single-Channel-Sys tem vgl. Dølvik in diesem Heft.
} 
versicherte Vollzeiterwerbstätige nicht überschreiten. Ferner können teilzeitversicherte Arbeitslose, die eine neue Beschäftigung unter 30 Wochenstunden annehmen, zusätzlich Arbeitslosenversicherungsleistungen in Anspruch nehmen (für die Dauer von 52 Wochen innerhalb der letzten 70 Wochen.$^{3}$

Um das effektive Arbeitsangebot von bisher Teilzeitbeschäftigten zu erhöhen, hat die Regierung vor Kurzem die Höchstdauer der zusätzlichen Transferleistungen auf 30 Wochen reduziert. Neben der Aufstockung der Löhne einiger Teilzeitbeschäftigter werden auch Zusatzleistungen ausgezahlt, um Arbeitnehmer in Branchen mit saisonaler Fluktuation zu halten.

Im Allgemeinen gelten für Teilzeitbeschäftigte die gleichen Kollektivverträge und Gesetze wie für Vollzeitbeschäftigte. Auch im Hinblick auf das kollektivvertragliche Rentensystem gehören sie demselben System an, allerdings mit proportional reduzierten Beiträgen und Leistungen. Zusammenfassend wurde Teilzeitarbeit zu einer institutionalisierten und regulierten Beschäftigungsform auf dem dänischen Arbeitsmarkt, die in vieler Hinsicht regulärer Vollzeitarbeit gleicht.

\subsection{BEFRISTUNG}

Eine weitere Form atypischer Beschäftigung, die befristete Beschäftigung, gewinnt in Europa an Bedeutung. Befristete Arbeitsverträge enden vereinbarungsgemäß zu einem bestimmten Zeitpunkt ohne weitere Ankündigungen, sei es ein fixiertes Datum, der Abschluss einer bestimmten Aufgabe oder die Rückkehr eines temporär vertretenen Arbeitnehmers (Danish Employers Association 1999, S. 204; Hasselbalch 2003). Der Anteil von Arbeitnehmern mit befristeten Verträgen blieb in Dänemark, abgesehen von einem leichten Rückgang von ungefähr 11 \% im Jahr 1996 auf 9 \% im Jahr 2007, über die Jahre hinweg weitgehend stabil, während der Anteil in den EU-15 Ländern in der gleichen Periode von $12 \%$ auf $15 \%$ anstieg. Die Verbreitung von befristeten Verträgen ist in Dänemark im Vergleich zu einer Reihe anderer EU-Staaten also eher gering.

Die wenigen vorliegenden Studien zu befristeten Arbeitsverträgen in Dänemark zeigen, dass die Wahrscheinlichkeit, einen befristeten Arbeitsvertrag zu bekommen, bei Frauen und niedrig qualifizierten Personen am größten ist (Eriksson/Jensen

Abb. 2: Regulierung zeitlich befristeter Beschäftigung (Index) - ausgewählte EU-Staaten 2003 -

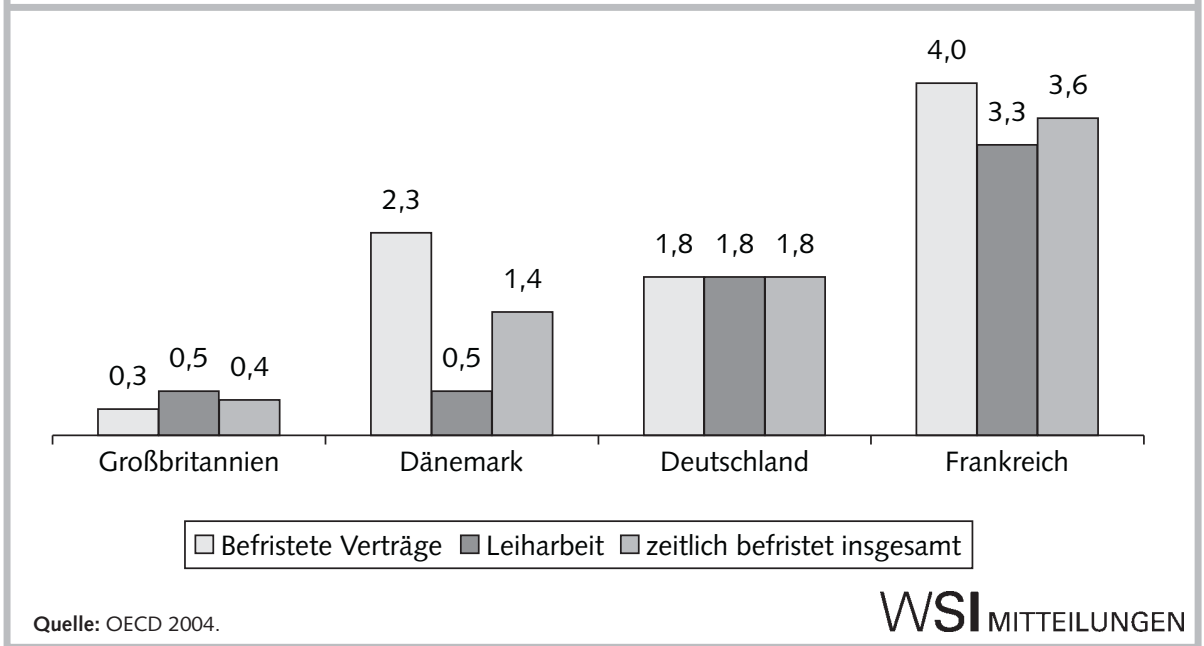

2003). Ungefähr ein Drittel der in befristeten Verträgen arbeitenden Personen wählt diese Beschäftigungsform, weil sie keine unbefristete Stelle finden (zwei Drittel hiervon Frauen) (Statistics Denmark 2004). Befristete Arbeitnehmer erhalten eine geringere Entlohnung als unbefristet Beschäftigte. Die Entlohnung weicht um $6 \%$ bis $7 \%$ ab (Eriksson/Jensen 2003, S. 13), bei einem höheren Risiko für Befristete, einen Niedriglohn, also weniger als $60 \%$ des Medianlohns, zu beziehen (Gash 2005). Zudem erhöht eine vorhergehende befristete Beschäftigung die Chancen, wieder eine befristete Anstellung zu erhalten (Eriksen und Jensen 2003), sodass die Wahrscheinlichkeit steigt, über einen längeren Zeitraum befristet beschäftigt zu sein.

Auch befristete Verträge werden durch Gesetze und Kollektivverträge reguliert. Mit Ausnahme der Kündigungsfrist werden befristete Arbeitnehmer im Allgemeinen von denselben Kollektivvereinbarungen und Gesetzen erfasst wie unbefristete Arbeitnehmer (beispielsweise bezüglich Urlaub, Seniorität, Krankengeld usw.).

Seit 2003 fallen alle befristet Beschäftigten unter das Gesetz zu befristeten Verträgen (Lov om Tidsbegronsede Ansattelser). Dieses Gesetz geht auf eine EU-Richtlinie aus dem Jahr 1999 zurück. Die Richtlinie verfolgte überwiegend das Ziel, die Qualität von befristeten Verträgen in allen EULändern zu verbessern, indem sichergestellt werden sollte, dass befristeten Arbeitnehmern dieselben Möglichkeiten und Rechte wie Arbeitnehmern in Normalarbeitsverhältnissen eingeräumt werden. So muss zum Beispiel befristeten Arbeitnehmern Zugang zur Weiterbildung zu densel- ben Konditionen wie unbefristet Beschäftigten gewährt werden. Außerdem sind die Arbeitgeber verpflichtet, befristete Arbeitnehmer über freie Stellen im Unternehmen zu unterrichten, um ihnen Chancen auf eine unbefristete Anstellung zu geben. Ein weiterer wichtiger Auftrag des Gesetzes war es, befristete Arbeitnehmer gegen missbräuchliche Kettenbefristungen zu schützen, indem Verlängerungen nur auf Basis objektiver Gründe wie Mutterschaftsurlaub, Krankheit oder Fertigstellung eines Projektes zulässig sind. In einigen Bereichen des Arbeitsmarktes (wie Lehre und Forschung) kann ein befristeter Vertrag nur zwei Mal verlängert werden.

Die OECD hat einen Index für die Stärke der Regulierung zeitlich befristeter Beschäftigung entwickelt (OECD 2004). Der Index misst die Beschränkungen in der Nutzung von befristeter Beschäftigung und Leiharbeit für Unternehmen im Hinblick auf den Einsatzbereich und ihre maximale Einsatzdauer (Abbildung 2).

Der Durchschnitt der Regulierungsstärke von Befristung und Leiharbeit liegt in Dänemark etwas unter dem EU-Niveau. Während die Stärke der Regulierung befristeter Verträge in Dänemark über dem Durchschnitt liegt, ist Leiharbeit weit unterdurchschnittlich reguliert. Diese Einstufung ergibt sich einerseits aus der erlaubten Höchstzahl der Verlängerungen als auch aus der kumulierten Höchstdauer (30 Mo-

3 Im Jahr 2006 haben ungefähr 12.000 Personen (Vollzeitäquivalente) zusätzliche Arbeitslosenversicherungsleistungen erhalten (Danish Employers Association 2007, S. 19) 
nate) befristeter Verträge, die zum Teil Gegenstand von Kollektivverträgen sind. ${ }^{4}$ Andererseits gibt es keine gesetzlichen Einschränkungen für den Einsatz von Leiharbeit und auch keine Beschränkungen für eine Wiederbeschäftigung von Leiharbeitern oder bezüglich ihrer Höchsteinsatzdauer (OECD 2004, S. 113f.).

Aus der Natur ihres Arbeitsvertrages folgt, dass befristet Beschäftigte eine geringere Arbeitsplatzsicherheit haben als unbefristete Arbeitnehmer. Wegen dieser immanenten Arbeitsplatzunsicherheit ist es für befristet Beschäftigte wichtig, Zugang zum sozialen Sicherungssystem zu erhalten, wenn sie arbeitslos werden. Hier entspricht das dänische System der Einkommenssicherung einem universalistischen Ansatz, der weder bei den Rechten noch bei den Pflichten zwischen Beschäftigungsformen unterscheidet. Allerdings erhalten nicht versicherte Arbeitslose staatliche Unterstützungsleistungen, während versicherte Arbeitslose Versicherungsleistungen aus dem Arbeitslosenversicherungsfonds bekommen. Die Anwartschaftsbedingungen für Arbeitslosenversicherungsleistungen können sich auf befristete Arbeitnehmer jedoch negativ auswirken, denn um Leistungen zu erhalten, muss man Mitglied eines Arbeitslosenversicherungsfonds sein und innerhalb der letzten drei Jahre mindestens 52 Wochen Mitgliedsbeiträge entrichtet haben.

\subsection{LEIHARBEIT}

Eine besondere Art der befristeten Beschäftigung ist die Leiharbeit. Sie weicht von einer Befristung im klassischen Sinne ab, weil die Zeitarbeitsagentur zwischen dem Entleiher und dem Leiharbeiter vermittelt und die Funktion des Arbeitgebers für Leiharbeiter übernimmt. Nach Eurofound (2006) war der Anteil der Leiharbeiter an allen Erwerbstätigen in der EU-15 in Dänemark am niedrigsten. Allerdings hat sich der Anteil der Leiharbeiter in Dänemark zuletzt verdreifacht und zwar von 0,3\% (1999) auf 0,9 \% (2006). Dieser Anstieg ist insbesondere auf den Wirtschaftsaufschwung seit 2004 und den Zuzug ausländischer Arbeitskräfte durch die EU-Erweiterung zurückzuführen (AE-Rådet 2006).

Leiharbeit wird bisher nicht gesetzlich, sondern durch Kollektivverträge reguliert. Traditionell standen die Gewerkschaften der Leiharbeit ablehnend gegenüber, da sie negative Effekte für das Lohnniveau und die Arbeitsbedingungen von Personen in Normalarbeitsverhältnissen fürchteten. Heute sind Leiharbeit und andere Formen "atypischer" Beschäftigung als dauerhafte Arbeitsmarktphänomene akzeptiert, die in das System der Kollektivvereinbarungen und -verhandlungen integriert wurden. Das führte dazu, dass die Leiharbeitsbranche größtenteils von Kollektivverträgen abgedeckt ist und Zeitarbeitsagenturen in großem Umfang auch Mitglied der Arbeitgeberverbände sind (Andersen 2007, S. 71).

Gleichwohl verbleibt eine Regulierungslücke außerhalb der Kollektivverträge, sodass diese Form der Regulierung langfristig wahrscheinlich nicht ausreichen wird. Im Juni 2008 hat sich der Rat der Europäischen Union in einer gemeinsamen Stellungnahme auf einen Vorschlag für eine Richtlinie zur Leiharbeit mit dem Ziel geeinigt, das Gleichbehandlungsprinzip sicherzustellen. Obwohl die dänischen Sozialpartner die Richtlinien innerhalb ihres Einflussbereichs umsetzen können, werden zur Abdeckung der restlichen Arbeitnehmer wohl zusätzliche Gesetze notwendig sein.

In verschiedenen Dimensionen von Sicherheit hat sich die Situation von Leiharbeitern in Dänemark zunehmend normalisiert: In Bezug auf Entlohnung, Rente, Urlaub, Krankengeld, Mutterschutz usw. entsprechen die Bedingungen weitgehend den Normalarbeitsverhältnissen. Wegen des komplexen Beschäftigungsverhältnisses kommt es jedoch immer noch zu Streitigkeiten darum, ob der Kollektivvertrag des Entleihbetriebs oder des Leiharbeitsunternehmens angewendet werden soll (und folglich, welche Bezahlung und Arbeitsbedingungen gelten). Außerdem stellt sich in Zukunft die Herausforderung, die Kollektivvereinbarungen auf die zunehmende Anzahl ausländischer Arbeitskräfte aus Ost- und Zentraleuropa auszuweiten (Andersen 2007).

\subsection{SOLO-SELBSTSTÄNDIGKEIT}

Solo-Selbstständige werden oft als eine Kategorie von Erwerbstätigen zwischen Arbeitnehmern und Arbeitgebern definiert, die sich durch das Merkmal auszeichnet, dass die Arbeit ohne weitere abhängig Beschäftigte ausgeführt wird (Madsen/Petersen 2000 , S. 66f.). In der Literatur wird Solo-Selbstständigkeit häufig als eine Form von ,atypischer" Beschäftigung bezeichnet, die Arbeitslose wegen mangelnder alternativer Beschäftigungsmöglichkeiten auf dem regulären Arbeitsmarkt ergreifen. Im Allgemeinen scheint das aber in Dänemark nicht der Fall zu sein, wo Solo-Selbstständigkeit vergleichsweise wenig verbreitet, leicht rückläufig und größtenteils Ergebnis einer freien Entscheidung ist.

Personen, die sich selbstständig machen, sind überdurchschnittlich gebildet, das heißt, die Mehrzahl von ihnen sind Facharbeiter oder verfügen über längere Ausbildungen (Erhvervs- og Byggestyrelsen 2006, S. 24). Selbstständigkeit ist am weitesten in den traditionellen Sektoren Landwirtschaft und Fischerei verbreitet, tritt aber auch im Baugewerbe und Dienstleistungsbereich häufiger auf, wo der Wechsel in die Selbstständigkeit als Karriereschritt für qualifizierte Arbeitskräfte gilt.

Selbstständigkeit wird nicht mehr als Strategie zur Vermeidung von Arbeitslosigkeit aufgefasst, und es gibt auch keine gezielten Programme mehr, Arbeitslose auf dem Weg in die Selbstständigkeit zu unterstützen. ${ }^{5}$ Einzig unter ausländischen Arbeitskräften, wo sonst fehlende von Qualifikationen oder Diskriminierung den $\mathrm{Zu}$ gang zu bezahlter Arbeit erschweren, wird Selbstständigkeit tatsächlich als alternativer Zugang zu Beschäftigung begriffen (Rezaei 2007).

Selbstständige haben - entsprechend der universalistischen Konzeption - Ansprüche auf dieselben Formen sozialer Sicherung (Arbeitslosenleistungen, Krankengeld und Mutterschaftsleistungen) wie abhängig Beschäftigte. In einigen Fällen gelten jedoch besondere Regelungen. Das betrifft die Arbeitslosenversicherung, wo Selbstständige und ihre mithelfenden Ehegatten freiwillig in zwei spezielle Arbeitslosenversicherungsfonds einzahlen können und somit Leistungen in dem Fall erhalten,

\footnotetext{
4 In einer Umfrage im Jahr 2000 haben Madsen und Petersen herausgefunden, dass $88 \%$ aller Arbeitnehmer mit befristeten Verträgen von Kollektivvereinbarungen erfasst wurden, $74 \%$ waren Mitglied des Arbeitslosenversicherungsfonds und $70 \%$ waren Mitglied einer Gewerkschaft (Madsen/Petersen 2000, S. 74, 76).

5 Frühere Versuche, die Selbstständigkeit als Beschäftigungsmöglichkeit für Langzeitarbeitlose zu fördern, wurden abgebrochen, weil sie nicht zu den erwarteten Ergebnissen geführt haben, denn die Mehrheit der Arbeitslosen, die an dem Programm teilnahmen, brachten nicht die Qualifikationen mit, die eine Selbstständigkeit erfordert (Ploughmann/Buhl 1998).
} 
dass sie ihr Geschäft aufgeben müssen. ${ }^{6}$ In ähnlicher Art und Weise können Selbstständige oder mithelfende Familienangehörige, die auch Anrechte auf staatliches Krankengeld (einschließlich Mutterschaftsgeld und Elterngeld) nach zweiwöchiger Krankheit haben, eine freiwillige Versicherung abschließen, die ihnen Ersatzleistungen ab dem ersten oder dritten Tag einer Krankheit oder Abwesenheit gewährt. Selbstständige und ihre Ehegatten haben im Prinzip die gleichen Rechte auf finanzielle Absicherung im Zusammenhang mit der Geburt eines Kindes wie abhängig Beschäftigte. Die Vorrausetzungen für Mutterschafts- und Elterngeld sind: In den letzten zwölf Monaten vor der Leistung müssen mindestens sechs Monate selbstständiger Tätigkeit liegen, mit einer wöchentlichen Arbeitszeit von mindestens der Hälfte einer normalen Vollzeitstelle (momentan 37 Stunden pro Woche); einer davon direkt vor der Auszeit. Zuzahlungen für den Einsatz einer Vertretung während des Mutterschaftsurlaubs oder der Elternzeit existieren nicht.

Obwohl eine Reihe von Initiativen mit dem Ziel gestartet wurde, das Unternehmertum gegenüber einer weit verbreiteten „Kultur der abhängigen Beschäftigung“ zu stärken, die Idee der Selbstständigkeit unter Schülern und Studenten zu popularisieren und Gründer zu beraten und zu unterstützen, ist die soziale Absicherung von Selbstständigen bisher kein Thema politischer Kontroversen. Das ist wahrscheinlich darauf zurückzuführen, dass Selbstständige vom allgemeinen „Sicherheitsnetz” des dänischen sozialen Sicherungssystems abgedeckt werden.

\subsection{FLEXJOBS}

Während die bisher erörterten Formen atypischer Beschäftigung annähernd die gleiche Regulierung bezüglich der Entlohnung und der Arbeitsbedingungen wie Normalarbeitsverhältnisse aufweisen, gibt es bei sogenannten Flexjobs wesentlich stärkere Abweichungen.

Flexjobs wurden 1998 als Teil eines Versuches eingeführt, den Zustrom in die Berufsunfähigkeitsrente zu bremsen. Flexjobs zielen auf Individuen mit langfristig eingeschränkter Arbeitsfähigkeit ab und sollen eine Alternative zur Berufsunfähigkeitsrente darstellen. Die Flexjobs werden mit dauerhaften Lohnzuschüssen subventioniert und sind sowohl im privaten als auch öf- fentlichen Sektor verfügbar. Wegen der eingeschränkten Arbeitsfähigkeit der Zielgruppe reduziert sich die Anzahl der Arbeitsstunden und/oder Aufgaben je nach Vereinbarung zwischen Arbeitgeber, der in Flexjobs Beschäftigten und der lokalen Behörde, die für die Administration des Programms zuständig ist. Die lokalen Behörden gewähren Arbeitgebern Lohnzuschüsse je nach Grad der reduzierten Arbeitsfähigkeit des Beschäftigten (Zweidrittel oder die Hälfte der Entlohnung), sodass die Personen mit Flexjobs unabhängig von ihrer wöchentlichen Arbeitszeit den vollen betriebsüblichen Lohn erhalten. Es liegen auch starke wirtschaftliche Anreize für die Verwaltung vor, Flexjobs für potenzielle Rentenbezieher zu finden, da die dänische Regierung für Flexjobs 50 \% der Zuschüsse erstattet und bei der Berufsunfähigkeitsrente nur $35 \%$.

Nach der Einführung der Flexjobs im Jahr 1998 hat sich ihre Zahl sehr stark erhöht und lag im Jahr 2006 bei 48.000 Personen (1,7 \% der Erwerbstätigen, $20 \%$ der Anspruchsberechtigten für Berufsunfähigkeitsrente). Das Programm wurde so beliebt, dass die Behörden, die für die Vermittlung von entsprechenden Arbeitsstellen zuständig sind, Schwierigkeiten wegen des großen Andrangs hatten. Eine zunehmende Anzahl von ehemaligen FlexjobBeschäftigten empfangen sogenannte Flexarbeitslosenleistungen (11.000 Personen im Jahr 2007), die 9\%-18\% niedriger liegen als die normalen Arbeitslosenleistungen. Als das Programm eingeführt werden sollte, sprachen sich die Gewerkschaften nämlich gegen die Aufnahme der FlexjobBeschäftigten in das Arbeitslosenversicherungssystem aus und konnten die Regierung davon überzeugen, ein paralleles, aber weniger abgesichertes und nicht so attraktives staatliches System für die Arbeitslosen- und Frühverrentungsleistungen solcher Beschäftigten einzurichten (Bredgaard 2004).

Es wird allerdings auf politischer Ebene zunehmend zur Kenntnis genommen, dass das Flexjobprogramm finanziell zu lukrativ für alle beteiligten Parteien - die in Flexjobs-Beschäftigten, die Behörden und die Arbeitgeber - geworden ist. Die Regierung hat deshalb ihre Absicht erklärt, das Programm auf solche Personen zu beschränken, die weder die Voraussetzungen für eine reguläre Beschäftigung noch für den Bezug einer Berufsunfähigkeitsrente erfüllen (Ministry of Finance 2007).

\section{Fazit und Schluss- folgerungen}

Es gibt kaum empirische Belege für die These, dass atypische Beschäftigung in Dänemark üblicher oder gar typisch wird. Vielmehr ist der Anteil von Selbstständigen und Befristeten in den letzten zehn Jahren sogar leicht zurückgegangen, während der Anteil von Teilzeitarbeitern in der letzten Zeit etwas zugenommen hat. Im Gegensatz zu einer Reihe von anderen europäischen Ländern ist der dänische Arbeitsmarkt weiterhin von einem dominierenden Kern in regulärer Vollzeit tätiger Arbeitnehmer mit unbefristeten Arbeitsverträgen und einer viel kleineren und relativ stabilen Randgruppe von atypischen Beschäftigungsverhältnissen gekennzeichnet. Hierzu zählen Teilzeitarbeit, Befristung, Leiharbeit, SoloSelbstständigkeit und (als eine dänische Besonderheit) Flexjobs.

Der vergleichsweise geringe Anteil von atypischen Beschäftigungsverhältnissen in Dänemark wird meist auf die liberale Gesetzgebung zum Kündigungsschutz zurückgeführt. Ein liberaler Kündigungsschutz tendiert dazu, die Dynamik des Arbeitsmarktes zu erhöhen, und der scheint die Risikoaversion der Arbeitgeber gegenüber der Einstellung regulär Beschäftigter $\mathrm{zu}$ vermindern. Das bedeutet, dass die Flexibilisierungsbedürfnisse der Arbeitgeber bei Einstellung und Entlassung mit normalen Beschäftigungsverhältnissen befriedigt werden können und dass Arbeitgeber nicht auf atypische Beschäftigungsformen ausweichen müssen, um extern numerisch flexibel zu sein.

Trotzdem können ungefähr $15 \%$ der Erwerbstätigen als ,atypisch“ Beschäftigte (in Selbstständigkeit, Befristung, Leiharbeit und Flexjobs) bezeichnet werden. Zusätzlich arbeiten ungefähr $25 \%$ der Beschäftigten in Teilzeit. Eine Unterkategorie der befristeten Beschäftigten, die zunimmt, bilden die Leiharbeitnehmer. Ihr Anteil hat sich in den letzten zehn Jahren verdreifacht, wenn auch von einem relativ niedrigen Ausgangsniveau aus.

\footnotetext{
6 Ungefähr 70 \% der Selbstständigen sind Mitglied eines Arbeitslosenversicherungsfonds, was nur etwas unter dem Durchschnitt der abhängig Beschäftigten liegt (Madsen/Petersen 2000, S. 68).
} 
Obwohl sich Teilzeitarbeit zu einer regulären und abgesicherten Form der Beschäftigung entwickelt hat, gaben einige Teilzeitbeschäftigte an, in dieser Beschäftigungsform festzustecken, weil sie keine Aussicht auf eine Vollzeittätigkeit hätten. Das Gleiche gilt für befristet Beschäftigte, da ein Drittel von ihnen angab, dass sie einen befristeten Arbeitsvertrag eingegangen sind, weil sie keine dauerhafte Anstellung finden konnten. Frauen und Personen mit niedrigem Bildungsniveau sind außerdem in Teilzeitarbeit und zeitlich befristeten Beschäftigungsformen überrepräsentiert. Selbstständigkeit scheint andererseits keinen „Fluchtweg” aus der Arbeitslosigkeit darzustellen, sondern beruht eher auf einer freiwilligen individuellen Entscheidung vielleicht mit Ausnahme einiger Gruppen von ausländischen Arbeitskräften.

Atypische Beschäftigung wird häufig mit niedriger Arbeitsplatzsicherheit, schlechten Arbeitsbedingungen und geringer Entlohnung, reduzierten Ansprüchen auf soziale Sicherungsleistungen, einem eingeschränkten Zugang zum Lebenslangen Lernen und zu Maßnahmen der aktiven Arbeitsmarktpolitik in Verbindung gebracht. Dies scheint insgesamt für Dänemark nicht zuzutreffen. Die diversen atypischen Beschäftigungsverhältnisse sind weitgehend durch die Kollektivverträge und Gesetze erfasst und genießen somit einen vergleichbaren Schutz wie Normalarbeitsverhältnisse. Man kann argumentieren, dass die dänische Flexicurity der regulären Arbeitnehmerschaft auf die atypischen Beschäftigten ausgeweitet wurde.

Der dänische Fall kann als Beispiel dafür dienen, dass die Entscheidung der Arbeitgeber, auf atypisch Beschäftigte zurückzugreifen, nicht nur von der Stärke des Kündigungsschutzes für regulär Beschäftigte beeinflusst wird, sondern auch von dem Ausmaß und der Ausgestaltung der sozialen Sicherung von irregulär Beschäftigten. Wenn atypisch Beschäftigte von den gleichen Gesetzen und Kollektivvereinbarungen wie gewöhnliche Arbeitnehmer geschützt werden, sind die Anreize für Ar- beitgeber, die Regulierung durch die Einstellung atypisch Beschäftigter zu umgehen - unter sonst gleichen Bedingungen -, gering. Folglich könnte anstatt der Lockerung der Kündigungsschutzbestimmungen für Normalarbeitsverhältnisse eine praktikable Politikstrategie in Europa auch lauten, die atypische Beschäftigung regulativ zu normalisieren, um der zunehmenden Segmentierung der Arbeitsmärkte entgegenzuwirken, die in einer Reihe von europäischen Staaten stattfindet. Als Nebeneffekt würde diese Möglichkeit zweifelsohne auch auf weniger politischen und sozialen Widerstand treffen und besser den Ansprüchen der Erwerbspersonen in Europa an eine individuelle Lebensführung gerecht werden.

Es gibt nach unserer Ansicht mindestens drei mögliche Erklärungen für die vergleichsweise niedrige Verbreitung von atypischer Beschäftigung in Dänemark. Erstens impliziert die Universalität des Systems des Einkommensschutzes, dass reguläre wie auch nicht standardisierte Erwerbstätige von der Arbeitslosenversicherung erfasst werden oder Sozialgeld zu fast gleichen Bedingungen erhalten können. Die relative Großzügigkeit der sozialen Sicherung erlaubt es den Arbeitnehmern, Angebote atypischer und insbesondere prekärer Beschäftigung abzulehnen. Zweitens sind die Gewerkschaften vergleichsweise stark (hohe Gewerkschaftsdichte, hohe Abdeckung mit Tarifverträgen und vorhandene Fähigkeit, bindende Vereinbarungen mit Vertretern der Arbeitgeberseite abzuschließen) und haben sich in der Vergangenheit für die Reduzierung ,atypischer" Beschäftigung eingesetzt. In letzter Zeit sind sie allerdings auch dazu übergegangen, die soziale Sicherung der „atypisch" Beschäftigten zu verbessern, um ihre Mitglieder zu schützen. Darüber hinaus haben die relativ hohen Mindestlöhne in Verbindung mit einer niedrigeren Arbeitsplatzsicherheit und hoher Beschäftigungsmobilität einen sehr wettbewerbsfähigen und produktiven Arbeitsmarkt geschaffen, auf dem im Gegensatz zu anderen europäischen Ländern für Arbeitgeber kaum Anreize existieren, auf „atypische“ Beschäftigung zurückzugreifen.

Allerdings ist die dänische Flexicurity auch mit einigen Herausforderungen konfrontiert, die nicht unerwähnt bleiben sollen. Erstens nimmt die Universalität des Systems der Einkommenssicherung ab. In den letzten Jahren sind die Sozialversicherungsleistungen für einige Gruppen (wie Ausländer) gekürzt worden. Außerdem wurde die Höhe der Arbeitslosenversicherungsleistungen seit den 1980er Jahren nicht mehr ganz der Preis- und Lohnsteigerungsrate angepasst. Das könnte die Großzügigkeit des Einkommensschutzes für spezifische Gruppen, wie Flüchtlinge und andere Ausländer oder auch Besserverdiener, infrage stellen. Im Zusammenhang hiermit verfolgt die aktive Arbeitsmarktpolitik zunehmend das Motto „Arbeit zuerst!“ anstelle von Qualifizierung, was insbesondere in der neuen Philosophie „jede Arbeit ist gute Arbeit” zum Ausdruck kommt. Die Möglichkeit, atypische oder prekäre Arbeit abzulehnen, wird zunehmend eingeschränkt. Zweitens verliert die Gewerkschaftsbewegung in Dänemark, wie in anderen europäischen Ländern auch, an Einfluss und könnte in Zukunft vielleicht nicht mehr genug Stärke aufweisen, das „dänische Modell” auf neue Formen von atypischer Beschäftigung auszuweiten, insbesondere in neuen Industriesektoren und im Hinblick auf die wachsende Zahl ausländischer Arbeitskräfte vor allem aus den neuen Beitrittsländern. Letztendlich können auch die individualisierteren Lebensläufe und -stile nicht mehr so leicht mit einem Normalarbeitsverhältnis in Einklang gebracht werden.

Wenn das politische Ziel ist, einen sicheren und flexiblen Arbeitsmarkt zu gewährleisten, wird die größte Herausforderung darin bestehen, regulierte Mobilität und reibungslose Übergänge zwischen verschiedenen Positionen innerhalb und auBerhalb des Arbeitsmarktes zu schaffen, anstatt unregulierter Mobilität und Beschäftigung ohne Zukunftsaussichten Vorschub zu leisten. 
Andersen, S. K. (2007): Vikarer mellem fleksibilitet og sikkerhed, Tidsskrift for Arbejdsliv 4, S. 63-79

AE-Rådet (2006): Historisk højt antal vikarer, Economic Council of the Labour Movement, http://www.aeraadet.dk/media/filebank/org/ vikarbesk-2006q3-mw.pdf

Auer, P./Cazes, S. (Hrsg.) (2003): Employment stability in an age of flexibility. Evidence from industrialized countries, International Labour Office, Geneva

Auer, P. (2007): In Search of Optimal Labour Market Institutions, in: Jørgensen, H./Madsen, P. K. (Hrsg.): Flexicurity and Beyond - Finding a New Agenda for the European Social Model, Copenhagen, S. 67-99

Bredgaard, Th. (2004): Virksomhedernes sociale ansvar - fra offentlig politik til virksomhedspolitik, Ph.d.-dissertation, Department of Economics, Politics and Administration, Aalborg University, Aalborg Bredgaard, T./Larsen, F./Madsen, P. K. (2005): The Flexible Danish Labour Market - A Review CARMA Research Paper 2005:01, Aalborg Bredgaard, T./Larsen, F./Madsen, P. K. (2006): Opportunities and Challenges for Flexicurity - The Danish Example, Transfer 1, S. 61-83 Danish Confederation of Trade Unions (2004): Deltidsloven med kommentarer, Copenhagen: LO

Danish Employers Association (1999): Arbejdsmarkedsrapport 1999,

Dansk Arbejdsgiverforening, Copenhagen

Danish Employers Association (2007): Arbejdsmarkedsrapport 2007, Dansk Arbejdsgiverforening, Copenhagen

Eurofound (2003): Part-time work in Europe, European Foundation for the Improvement of Living and working Conditions, Dublin

Eurofound (2006): Temporary agency work in an enlarged European Union, European Foundation for the Improvement of Living and working Conditions, Dublin

European Commission (2006): Employment in Europe 2006, Brussels European Commission (2007a): Towards Common Principles of Flexicurity: More and Better Jobs through flexibility and Security, Communication $\operatorname{COM}(2007) 359$, Brussels

European Commission (2007b): Employment in Europe 2007, Brussels Erhvervs- og Byggestyrelsen (2006): Iværksættere og nye virksomheder, Nøgletal

Eriksson, T./Jensen, P. (2003): Tidsbegränsade anställingar - danske erfarenheter, Working Paper 03-15, Department of Economics, Aarhus School of Business

Expert Group on flexicurity (2007): Flexicurity Pathways - turning hurdles into Stepping Stones, Brussles June

Gash, V. (2005): The labour market outcome of atypical employment in Ireland and Denmark. Combat Poverty Agency, Working paper 05/3 Hasselbalch, O. (2003): Tidsbegrænset ansættelse, Jurist- og Økonomforbundets Forlag, Copenhagen
Jørgensen, H./Madsen, P. K. (2007): Flexicurity and Beyond - Reflections on the Nature and Future of a Political Celebrity, in: Jørgensen, H./ Madsen, P. K. (Hrsg.): Flexicurity and Beyond - Finding a New Agenda for the European Social Model, Copenhagen, S. 7-39

Kalleberg, A. L. (2000): Non-standard Employment Relations: Part-time, Temporary and Contract work, in: Annual Review of Sociology, S. 341365

Madsen, M./Petersen, A. (2000): Nye ansættelsesformer på det danske arbejdsmarked - bliver de atypiske de typiske?, Copenhagen: Danish Confederation of Trade Unions, LO-dokumentation 2

Madsen, P. K. (2003): "Flexicurity" through labour market policies and institutions in Denmark', in: Auer, P./Cazes, S. (Hrsg.): Employment stability in an age of flexibility. Evidence from industrialized countries, Geneva, ILO, S. 59-105

Madsen, P. K. (2004) The Danish model of "flexicurity": experiences and lessons, Transfer 2, S. 187-207

Madsen, P. K. (2005) The Danish road to flexicurity: Where are we. And how did we get there?, in: T. Bredgaard and F. Larsen (Hrsg.): Employment policy from different angles, Copenhagen

Madsen, P. K. (2006): How can it possibly fly? The paradox of a dynamic labour market in a Scandinavian welfare state, in: Campbell, J. L./Hall, J. A./Pedersen, O. K. (Hrsg.): National Identity and a Variety of Capitalism: The Case of Denmark, Montreal, S. 321-355

Ministry of Finance (2007): Mod nye mål - Danmark 2015, Regeringen:

Finansministeriet, august

Ministry of Labour (1999): Arbejdsmarkedsreformerne - en status, Arbejdsministeriet, København

Nedergaard, P. (2004): Den danske model I et integreret Europa, Jurist- og Økonomforbundets Forlag, Copenhagen

OECD (2004) Employment Outlook 2004, Paris

Ploughmann, P./Buhl, S. (1998): Self-employment in Denmark - Trends and policy, Paper presented at Conference on Self-employment, Burlington/Canada, September

Rezaei, S. (2007): Breaking out: The Dynamics of immigrant owned businesses, in: Journal of Social Sciences 3, S. 94-105

Statistics Denmark (2004): Nyt fra Danmarks Statistik 213, 19 May, Danmarks Statistik, Copenhagen

Wehner, C./Johansen, M. M./Navrbjerg, S. (2002): Deltidsarbejde i Danmark, Copenhagen: FAOS Information 035 Wilthagen, T. (1998) Flexicurity - A new paradigm for labour market policy reform?, WZB Discussion Paper, FSI, 98-202, Berlin Wilthagen, T./Tros, F. (2004) The concept of "flexicurity": A new approach to regulating employment and labour markets, in: Transfer 2 , S. 166-187 